\title{
Further Cytological Studies on Haploid and Diploid Strains of Tetrahymena pyriformis with Special Reference to Univalent Spindles
}

\author{
Alfred M. Elliott and Gordon M. Clark \\ Department of Zoology, University of Michigan, Ann Arbor, Michigan, U.S.A. \\ Received April 30, 1957
}

In insects with male haploidy, it is usual for the first meiotic division to be completely suppressed so that sperms are formed with the same number of chromosomes as the somatic tissues of the adult male. Vestiges of the first meiotic division do occur, however, in some cases. In the male bee (Apis mellifica L.), the prophase stages of the first meiotic division take place, but the metaphase and anaphase are entirely lacking; the second meiotic division then follows (Nachtsheim 1913); in some other haploid insects the first division is entirely suppressed (Hughes-Schrader, 1930). In plants, however, where haploid sporophytes do occur occasionally, both meiotic divisions usually are found. In true haploids all the chromosomes behave as univalents, dividing in either the first or second division, but never in both divisions. Some haploid plants have small sections of the chromosomes reduplicated in other members of the chromosome sets; in these cases the small reduplicated sections may pair at zygotene and form chiasmata later, so that a few bivalents are found at the first meiotic division (Catcheside 1932).

In the protozoa, haploid forms have been derived by mating X-irradiated and non-irradiated cells of Paramecium aurelia (Kimball and Gaither 1955) and T. pyriformis (Elliott and Clark 1956). The cytology of conjugating diploid strains of $T$. pyriformis $(2 n=10)$ has been described by Elliott and Hayes (1953), and by Ray (1956). A preliminary report of cytological studies in crosses involving haploids and diploids has also been reported (Elliott and Clark 1956). It was during the course of more detailed cytological study of meiosis in haploid and diploid strains that the authors observed the occurrence of univalent spindles. The purpose of the paper is to report these findings.

\section{Materials and methods}

Only clones belonging to variety 2 were used in this investigation. Two haploid strains, TC95h/5 and UM351h/2 (mating types XI and IV respectively),

* This investigation was supported in part by a research grant (PHS G 3588) from the National Institutes of Health, Public Health Service. 
and a diploid strain, UM569 (mating type VIII), were used in these studies. The organisms were maintained in $1 \%$ proteose-peptone at $\mathrm{pH} 7.2$. For mating experiments cells were taken in the log phase of growth, washed three times in glass distilled water, starved for 24 hours, and mixed in depression slides. Matings were made between the two haploids, and between haploid UM351h/2 and the diploid clone. The organisms were maintained at $30^{\circ} \mathrm{C}$. throughout the entire mating process.

When conjugation was first noted samples were removed, prefixed in Nissenbaum's fluid for 15 seconds, and then fixed in Carnoy's $(2: 1)$ for 30 minutes, hydrated to $70 \%$ alcohol and stored for future use. Samples were removed at half-hour intervals up to and including the time the anlagen stages were visible.

Material for cytological observations was subjected to mild acid hydrolysis, stained in iron-acetocarmine two minutes and steamed to facilitate flattening of the cells for photographic purposes. Coverslips were sealed around the edges with a mixture of paraffin and beeswax (Ray 1956a).

A Bausch and Lomb phase contrast-microscope, equipped with $43 \times$ and $97 \times$ objectives and $10 \times$ oculars, was used in the examination of fixed preparations. Photomicrographs were made using Kodak $M$ plates $\left(4^{\prime \prime} \times 5^{\prime \prime}\right)$ at magnifications of $430 \times$ and $970 \times$ on the negative.

\section{Results and discussion}

In crosses of haploids and diploids, the diploid mate undergoes meiosis in the first two prezygotic divisions. The micronucleus migrates anterior to the macronucleus, enlarges, forms an elongated crescent and reaches a stage comparable to a pachytene-diplotene stage at maximum elongation of the crescent. As the crescent shortens, five bivalent chromosomes can be counted as they proceed into metaphase of the first division. In succeeding divisions, a haploid migratory nucleus is deposited in the haploid member of the pair, the results of which leads to the development of the anlagen stages in both members of the pair from the hemicaryon (Elliott and Clark 1956).

Meiosis in the haploid when involved in crosses with other haploids or diploids is extremely variable. The crescent never develops beyond a two thread stage and no pachytene-diplotene stage is realized (Pl. XVII, Figs. 1-3). As the crescent shortens, five univalent chromosomes can be counted which may ; (1) segregate at random at Metaphase I, (2) round up to form a singlere stitution nucleus, or (3) undergo complete disintegration (Pl. XVII, Figs. 4, 7 and 8). In a small number of cases, following the formation of the crescent the following phenomenon is observed. The univalents round up to form five individual nuclei, each of which is associated with a small spindle. Elongation of the spindle takes place and the univalent chromosome migrates to one or the other pole (Pl. XVII, Fig. 5). These univalents apparently then disintegrate 

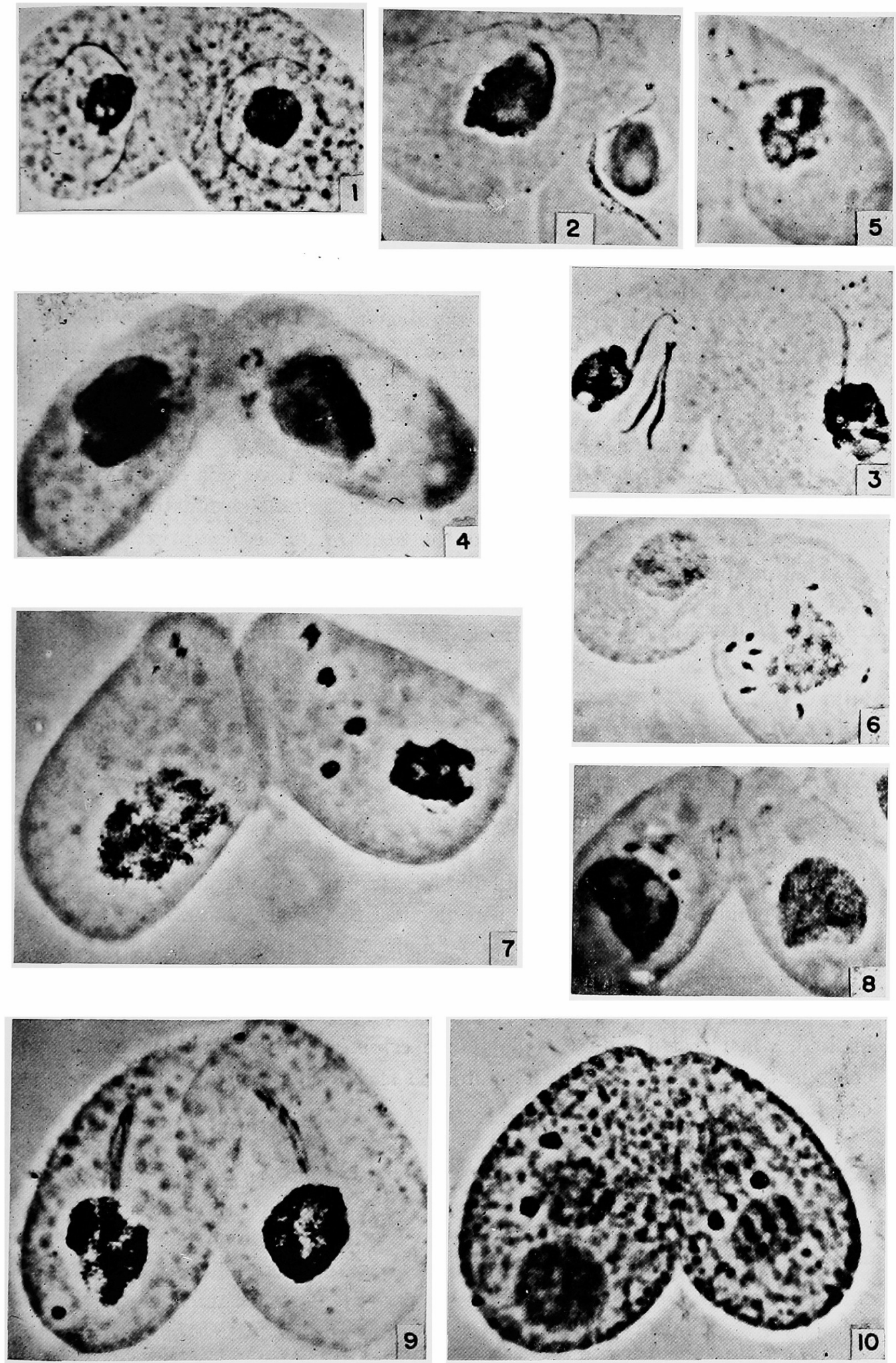

A. M. Elliott and G. M. Clark: Cytological studies in Tetrahymena 
or fuse to form one or more restitution nuclei which attempt at a later stage to undergo a second division.

This same result has been observed in another haploid which contains two micronuclei. During meiosis two crescents form resulting in 10 univalent spindles (Pl. XVII, Fig. 6). This phenomenon has never been observed during conjugation of diploids in variety two, but has been noted in variety five (Ray, personal communication). More than one crescent, however, has been seen in multi-micronucleate strains (Pl. XVII, Fig. 3).

As far as can be ascertained, this is the first report of this phenomenon in ciliated protozoa. Cooper (1941) has demonstrated that during mitosis in the mite, Pediculopsis, each chromosome constitutes a miniature nucleus or karyomere, the wall of which disappears rather late in mitosis. The orientation of chromosomal fibers to form a bipolar spindle in the egg of the insect Acroschismus (Hughes-Schrader 1924) resembles the events reported here. Our observations reveal that the centromeres have not divided in the first prezygotic division since no separations of the half-chromatids constituting the univalent is observed and that the centromere divides previous to the second division. It would also seem that the univalents are arranged linearly along the prophase crescent.

In matings with diploids the usual consequence of meiosis in the haploid is the complete break down of the products of meiosis with no genetic contribution being made by the haploid member of the pair. The diploid undergoes a mitotic third prezygotic division (Pl. XVII, Fig. 8) with a haploid migratory nucleus being deposited in the haploid. Both conjugants now contain single haploid nuclei which are genetically identical. Two successive postzygotic divisions occur resulting in the formation of macronuclear anlagen and new micronuclei (Pl. XVII, Figs. 9, 10). It should be noted however, that upon formation of the new nuclear components no old macronucleus is found. In matings of two diploids the old macronucleus persists for several hours after the conjugants have separated and disappears finally just previous to the first fission.

The cytological picture during meiosis in which both conjugants are haploid is quite erratic. The nuclear events already outlined above for the haploid, when mated with the diploid, is essentially the same, when the two conjugants are haploid at least up to the stage where nuclear transfer from one mate to the other should occur.

No nuclear transfer from one haploid to the other has been observed. Chromatin fragments, resulting from abortive prezygotic divisions, migrate to the region of attachment of the two conjugants and in a position where nuclear transfer normally occurs. These fragments probably then reunite to form restitution nuclei which in turn give rise to macronuclear anlagen and micronuclei. In conjunction with this process the old macronucleus disappears, some of the products of which most probably contribute to the formation of the new nuclear components. 
Viability in these crosses is poor $(0.5 \%)$ and the majority of animals die in the paired condition or if separation occurs death occurs after one or two fissions. Apparently only those animals in which a complete genome is present give rise to clonal cultures. This indeed is a rare event based on the fact of poor viability.

Future work with $\mathrm{C}$-mitotic agents may prove interesting and may, if successful, yield diploid homozygous lines for use in genetic analysis circumventing the need for finding methods of inducing autogamy which so far have not been fruitful.

\section{Literature cited}

Catcheside, D. G. 1932. The chromosomes of a new haploid Oenothera. Cytologia 4: 68. Cooper, K. W. 1941. Visibility of the primary spindle fibers and the course of mitosis in the living blastomeres of the mite, Pediculopsis graminum. Reut. Proc. Nat. Sci. 27: $480-483$.

Elliott, A. M. and Hays, R. E. 1953. Mating types in Tetrahymena. Biol. Bull. 105: 269284.

- and Clark, G. M. 1956. The induction of haploidy in Tetrahymena pyriformis following X-radiation. J. Protozool. 3: 181-188.

Hughes-Schrader, S. 1924. Reproduction in Acroschimus. J. Morph. 39: 157-207.

- 1930. The cytology of several species of iceryine Coccids with special reference to parthenogenesis and haploidy. Ibid. 50: 475.

Kimball, R. F. and Gaither, N. 1955. Behavior of nuclei at conjugation in Paramecium aurelia I. Effect of incomplete chromosome sets and competition between complete and incomplete nuclei. Genetics 40: 878-889.

Nachtscheim, H. 1913. Cytologische Studien über die Geschlechtsbestimmung bei der Honigbiene (Apis mellifica L.). Arch. Zellf. 11: 169.

Ray, C. 1956. Meiosis and nuclear behavior in Tetrahymena pyriformis. J. Protozool. 3 : $88-97$.

- 1956a. Preparation of chromosomes of Telrahymena pyriformis for photomicrography. Stain Tech. 31: 271-274.

\section{Explanation of Plate XVII}

Figs. 1-10. Photomicrographs made from acetocarmine preparations of haploid and diploid strains of $T$. pyriformis in conjugation. 1, formation of the crescent in conjugating haploids. 2, haploid at left showing crescent in the two-thread stage with the diploid at the right in the four-thread stage. 3, diploid at left showing formation of three crescents in contrast to the haploid at the right exhibiting a crescent in the two-thread stage. 4, haploid at left showing five univalent chromosomes with the diploid at the right showing five bivalents. 5, haploid member of a pair showing five univalents in association with their individual spindles. 6, haploid at left completely devoid of chromatin with the haploid at the right forming ten spindles. 7 , haploid at left with five univalents which will form a single restitution nucleus with the diploid at the right having completed the second prezygotic division. 8, diploid at left has undergone third prezygotic division. Notice five chromosomes with median to submedian centromeres. This haploid nucleus will be transferred to the haploid at the right. Notice disintegration of restitution nucleus in the haploid. 9, nuclear transfer has occurred and the first postzygotic division is in progress. 10, upon completion of second postzygotic division the macronuclear anlagen and new micronuclei are formed. Note absence of old macronucleus. 